\title{
SPONTANEOUS FLUCTUATIONS IN GLUCOSE CONTENT OF THE HEPATIC VENOUS BLOOD IN RESTING NORMAL HUMAN BEINGS
}

\author{
By PHILIP K. BONDY \\ (From the Medical Service, Grady Memorial Hospital, and the Department of Medicine, Emory \\ University School of Medicine, Atlanta, Georgia)
}

(Submitted for publication October 15, 1951 ; accepted November 29, 1951)

There is ample evidence that the liver helps the fasting animal to maintain the blood glucose concentration at normal levels by secreting glucose into the hepatic vein. After removal of the liver from experimental animals, hypoglycemia develops rapidly (1). In animals the concentration of glucose in the hepatic vein is usually higher than in the portal vein or the arteries (2). The application of the technique of venous catheterization has made it possible to show that similar relationships usually are found in the normal, unanesthetized human subject $(3,4)$.

It has usually been assumed that, under basal conditions, the secretion of glucose into the blood stream by the liver is a continuous process, and consequently, a fair estimate of the rate of release of glucose can be obtained by multiplying the difference between the arterial and hepatic venous glucose concentrations (A-HV) by the estimated hepatic blood flow (EHBF). The observations set forth in this paper indicate that the rate of release of glucose by the liver is highly variable, and that even the examination of a large number of A-HV pairs may fail to produce a reliable estimate of the rate of production of glucose by the liver.

\section{METHODS}

The subjects were 11 normal male volunteers convalescent from various diseases (Tables III and V). Most of the subjects were sedated with $0.5 \mathrm{gm}$. sodium amytal intramuscularly 30 minutes before the procedure and were drowsy but responsive when the samples were obtained. The intravenous catheter was inserted into the right antecubital vein and guided under direct fluoroscopic control into a right hepatic vein. Its position was adjusted until a free reflux of blood could be obtained (more than $10 \mathrm{ml}$. per minute). A Cournand needle was placed in the left brachial or femoral artery. In most subjects, a priming dose of $150 \mathrm{mg}$. bromsulfalein was immediately given intravenously, and following this a solution of bromsulfalein was infused intravenously, using an electric pump to deliver a constant quantity of the dye.
At the end of the experiment, a five-minute collection of dye from the pump was analyzed for bromsulfalein and the rate of infusion calculated from this figure. The rate of infusion was between 2 and $3 \mathrm{mg}$. per minute.

A period of 30 minutes was permitted to elapse after the start of the bromsulfalein injection. During this time the patient was reassured that no further operative procedure was contemplated, and most patients went to sleep.

After 30 minutes, the catheter was cleared of saline before sampling by withdrawing $5 \mathrm{ml}$. of blood, which were discarded. Specimens were then drawn simultaneously from the arterial needle and hepatic venous catheter. Five $\mathrm{ml}$. were preserved in fluoride-oxalate mixture, and analyzed for glucose. The copper tungstate filtrates (5) for glucose analysis were made within two hours after drawing the blood. The remainder of the blood was permitted to clot, and the serum analyzed for bromsulfalein. The procedure was repeated for additional samples at the time intervals indicated in the tables.

In most patients, the hepatic blood flow was estimated by the method of Bradley, Ingelfinger, Bradley and Curry (6). Splanchnic glucose balances were estimated by multiplying A-HV by EHBF.

\section{GLUCOSE ANALYSIS}

The detection of significant differences between arterial and hepatic venous glucose concentrations is limited by the accuracy of the glucose method. The error of the method, in turn, is a composite of a number of different errors. In order to estimate the accuracy of the Nelson method (7) and to assign the possible sources of error their proper importance, a series of tests was done.

It was found that the color developed by the addition of the arsenomolybdate solution at room temperature increases gradually to a plateau, which is not reached for 120 minutes. The increment is small, representing only a $5 \%$ increase; but a variation of $5 \%$ may be greater than the value of A-HV. All samples were therefore permitted to stand for 120 minutes or more before they were read in a Coleman Jr. spectrophotometer at $650 \mathrm{~m} \mu$.

An attempt was made to discover whether variations in the technique of protein precipitation cause any significant error in the method. Six separate protein precipitations (5) were made from a single, well-mixed sample of blood. In three, Ostwald pipettes were permitted to drain for 30 seconds after they had completely discharged their 
TABLE I

Analysis of variance of the Nelson glucose method Quadruplicate determinations in multiple protein-free filtrates prepared from the same blood specimen.

\begin{tabular}{c|c|c|c|c|c|c}
\hline \hline Filtrate No. & 1 & $2 *$ & 3 & $4^{*}$ & 5 & $6^{*}$ \\
\cline { 2 - 6 } mg./100 ml. & 62.0 & 61.9 & 61.9 & 61.8 & 62.0 & 61.2 \\
& 62.0 & 62.1 & 61.2 & 61.8 & 61.8 & 61.5 \\
\hline Mean & 61.4 & 61.1 & 61.0 & 61.2 & 61.5 & 62.0 \\
\hline
\end{tabular}

* $1 \mathrm{ml}$. Ostwald pipettes drained completely and then rinsed out.

$$
\begin{aligned}
\text { Group variance } & =0.1620 \\
\text { Individual variance } & =0.2038 \\
F & =0.7948
\end{aligned}
$$

Variance within filtrates is greater $(p<.01)$ than between filtrates.

contents of blood, and then were blown out once. In three others, the blood was permitted to drain into distilled water in a centrifuge tube, and the pipettes were then rinsed out thrice with the distilled water. Copper sulfate and sodium tungstate were added from volumetric pipettes, and the distilled water was measured with a calibrated burette. The results, shown in Table I, were submitted to an analysis of variance (8), which shows that there is greater variation from one tube to another in the same filtrate than there is between various filtrates. It made no difference whether the pipette was blown out or rinsed out. Consequently, the pipettes were rinsed out routinely, since this proved to be more rapid. All pipettes were calibrated by weighing the quantity of distilled water discharged under standard conditions. The maximum variation among pipettes was less than $1 \%$.

The analysis of variance proved that no advantage was gained by making multiple protein-free filtrates from the same sample. It was necessary, however, to estimate the number of replicates from each protein-free filtrate which had to be analyzed in order to afford the degree of accuracy desired. Table II shows the relationship between the number of replicates and the minimum difference detectable between two samples, with a $p=.02$. On the basis of these data, quadruplicate analyses were performed on

TABLE II

Effect of varying the number of replicates on the minimum detectable difference between two samples of glucose (Nelson method)

Based on data in Table I. Mean glucose concentration $=61.7 \mathrm{mg} . / 100 \mathrm{ml}$.

\begin{tabular}{c|c|c|c|c}
\hline \hline $\begin{array}{c}\text { No. of } \\
\text { replicates }\end{array}$ & $\begin{array}{c}\text { Degrees of } \\
\text { freedom }\end{array}$ & $\begin{array}{c}\text { Standard } \\
\text { error of the } \\
\text { difference } \\
\text { (mg./100 ml. })\end{array}$ & $\begin{array}{c}\text { Difference } \\
\text { for } \\
\text { fo. } \\
(\mathbf{m g} . / 100 \text { ml. })\end{array}$ & $\begin{array}{c}\text { Difference: } \\
\text { \% of mean }\end{array}$ \\
\hline 2 & 2 & 0.44 & 3.1 & 5.0 \\
3 & 4 & 0.36 & 1.4 & 2.3 \\
4 & 6 & 0.31 & 1.0 & 1.6 \\
5 & 8 & 0.28 & 0.8 & 1.3 \\
\hline
\end{tabular}

all protein-free filtrates. This made it possible to detect a difference of $1.6 \mathrm{mg} . / 100 \mathrm{ml}$. between samples in the range of $100 \mathrm{mg}$. glucose $/ 100 \mathrm{ml}$., with a probability of $2 \%$.

\section{RESULTS}

A-HV pairs were drawn at 10-minute intervals in four subjects (Table III). During this time, considerable variation occurred in both arterial and hepatic venous glucose concentrations. In three of the four subjects, the hepatic venous blood contained less glucose than the arterial on at least one occasion. In these subjects, the variations in A-HV difference and in splanchnic balance were so great that the mean values were not significantly different from zero. In two instances the mean values for $\mathrm{A}-\mathrm{HV}$ indicated a net retention of glucose by the liver, although this apparent retention was not significant when analyzed statistically.

Further insight into the rate of change of A-HV was sought by obtaining specimens at two-minute intervals in three of the subjects who had also been studied at 10-minute intervals (Table IV). The variations of $\mathrm{A}-\mathrm{HV}$ were even more erratic when examined at this short time interval than when observed at 10 -minute periods. The mean value for A-HV was negative in each case, indicating that the liver was releasing glucose into the systemic circulation, but the variation was so great that the mean was not significantly different from zero in any case.

These observations made it clear that the determination of isolated sets of $\mathrm{A}-\mathrm{HV}$ pairs gives an unreliable picture of the overall splanchnic glucose balance. An attempt was therefore made to estimate the mean A-HV difference by taking a continuous five-minute sample. It was hoped that this would make possible a direct integration of the difference between the arterial and hepatic venous glucose concentration curves.

The method of continuous sampling was as follows: The subject was given $2.5 \mathrm{ml}$. (25 mg.) heparin solution through the catheter immediately after the arterial needle had been placed. Since the object of the experiment was simply to follow A-HV differences, no estimation of hepatic blood flow was attempted. After 20 minutes, a length of plastic tubing approximately $10 \mathrm{~cm}$. in length was attached to the Cournand needle and closed off with a pinchcock. A three-way stopcock was attached to the catheter and the I.V. saline drip which serves to keep the catheter clear was disconnected. Using a $10 \mathrm{ml}$. syringe, blood was continually aspirated from the catheter at a rate of $1 \mathrm{ml}$. every five seconds. At the end of 25 


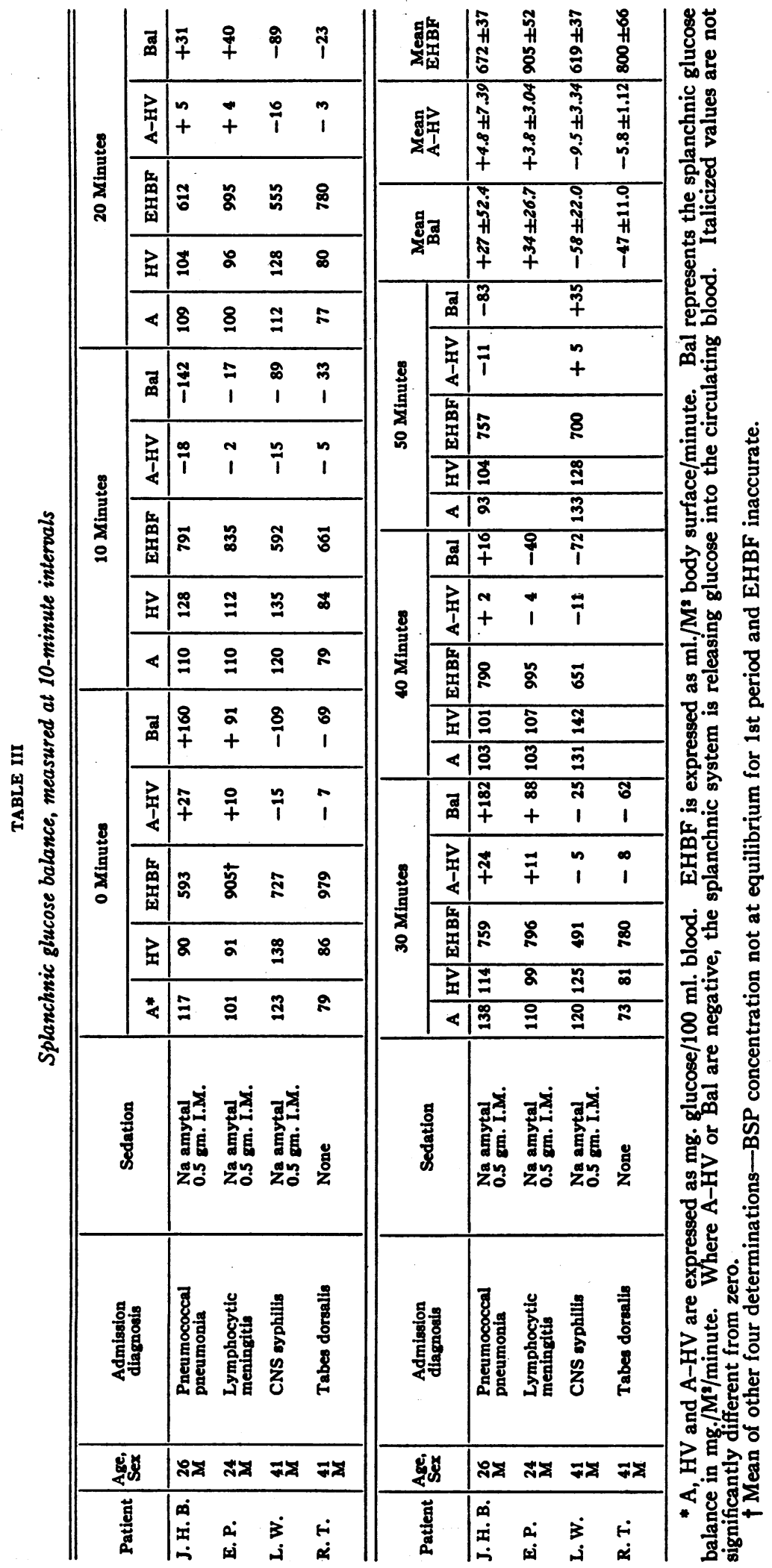


TABLE IV

Arterio-hepatic venous glucose differences, measured at two-minute intervals

\begin{tabular}{|c|c|c|c|c|c|c|c|c|c|c|c|c|c|c|c|c|c|c|c|c|}
\hline \multirow{2}{*}{ Patient } & \multicolumn{3}{|c|}{ o Minutes } & \multicolumn{3}{|c|}{2 Minutes } & \multicolumn{3}{|c|}{4 Minutes } & \multicolumn{3}{|c|}{6 Minutes } & \multicolumn{3}{|c|}{8 Minutes } & \multicolumn{3}{|c|}{10 Minutes } & \multirow{2}{*}{ Mean } & \multirow{2}{*}{$\begin{array}{c}\text { Standard } \\
\text { error }\end{array}$} \\
\hline & $\mathbf{A}^{*}$ & HV & A-HV & $\mathbf{A}$ & $\mathrm{HV}$ & A-HV & $\mathbf{A}$ & $\mathrm{HV}$ & A-HV & $\mathbf{A}$ & HV & A-HV & $\mathbf{A}$ & HV & A-HV & $\mathbf{A}$ & HV & A-HV & & \\
\hline $\begin{array}{l}\text { J. H. B. } \\
\text { E. P. } \\
\text { L. W. }\end{array}$ & $\begin{array}{r}77 \\
101 \\
126\end{array}$ & $\begin{array}{l}105 \\
103 \\
141\end{array}$ & $\begin{array}{l}-28 \\
-2 \\
-15\end{array}$ & $\begin{array}{r}77 \\
99 \\
158\end{array}$ & $\begin{array}{r}106 \\
99 \\
132\end{array}$ & $\begin{array}{r}-29 \\
0 \\
+26\end{array}$ & $\begin{array}{l}98 \\
98\end{array}$ & $\begin{array}{r}91 \\
118\end{array}$ & $\begin{array}{l} \pm 7 \\
-20\end{array}$ & $\begin{array}{r}83 \\
94 \\
113\end{array}$ & $\begin{array}{r}97 \\
99 \\
134\end{array}$ & $\begin{array}{l}-14 \\
-5 \\
-21\end{array}$ & $\begin{array}{l}131 \\
103 \\
121\end{array}$ & $\begin{array}{l}117 \\
105 \\
141\end{array} \mid$ & $\begin{array}{l}+14 \\
-2 \\
-20\end{array}$ & $\begin{array}{l}110 \\
110 \\
120\end{array}$ & $\begin{array}{l}128 \\
112 \\
135\end{array}$ & $\begin{array}{l}-18 \\
-2 \\
-15\end{array}$ & $\begin{array}{l}-11 \\
-5 \\
-9\end{array}$ & $\begin{array}{l} \pm 7.4 \\
\pm 3.0 \\
\pm 8.8\end{array}$ \\
\hline
\end{tabular}

* See footnote to Table III.

seconds, the stopcock was turned and the blood ejected from the side-arm into a fluoride-oxalate bottle. The same syringe was then used to draw the next sample in the same manner. The arterial sample was collected by permitting the blood to run freely from the plastic tube, using the pinchcock only to permit the receptacle to be changed every 30 seconds. A total of 10 pairs of specimens, representing a total of five minutes of continuous sampling, was obtained in each subject.

The individual samples were analyzed for glucose; and in addition integrated samples, representing the entire five-minute period, were made by pooling $1 \mathrm{ml}$. aliquots from all of the arterial and all of the hepatic venous specimens.

The results of the 30-second sampling are shown in Table V. The arterial glucose concentrations were subject to slight but statistically significant fluctuations. These variations, however, were small in comparison with the alterations in the glucose concentration in hepatic venous blood. In some instances, this variation still does not alter the consistently negative pattern of A-HV (J. B., J. L. T.) ; but in other cases periods of significant glucose retention were seen (V. H. $120^{\prime \prime}$ to $180^{\prime \prime}$; J. C. $210^{\prime \prime}$ to $240^{\prime \prime}$ ). The last columns of Table $\mathrm{V}$ compare the results of graphic integration of the A-HV difference, using a polar planimeter, with "chemical integration," using the mixed samples. For reference, the mean \pm standard error of the A-HV difference are also shown. The graphic integration and the arithmetic mean of the A-HV values are in good agreement. The mixed samples, however, do not agree well with the other two. The cause of this discrepancy is not immediately apparent. It probably represents the fact that the analytic error for the mixed sample is increased by the errors of pipetting the aliquots, mixing, etc. Moreover, errors in the $10 \mathrm{~A}-\mathrm{HV}$ pairs would be expected, on the basis of probability theory, to be distributed around zero, and therefore to tend to cancel out, whereas no such correc- tion occurs in the single set of chemical determinations performed on the mixed sample. It is quite clear therefore that chemical integration by mixing is much less accurate than the determination of multiple A-HV pairs. The same is doubtless true if a single pair of samples is drawn by constant sampling for five minutes. But even when multiple pairs are analyzed, the spontaneous variation of the blood glucose concentration is so great as to cast doubt on the significance of the estimation of splanchnic balances from these data. This is illustrated in the table where the $95 \%$ limits of confidence are listed for the four patients. This column gives for each patient the range within which the mean of the A-HV differences will fall $95 \%$ of the time if the experiment is performed repeatedly, assuming that the variability of blood glucose concentration does not change. From this column it can be seen that even when 10 pairs of samples are taken, the mean value of $\mathrm{A}-\mathrm{HV}$ cannot be defined with surety.

When the values for A-HV are followed, it is apparent that three of the four subjects had short periods of greatly accelerated glucose output (J. C. $30-60$; V. H. 0-30, 60-90; J. L. T. 90-150). No cause for these sudden "spikes" of glucose release is apparent. In view of the well-known effects of emotion and stress on the blood glucose concentration (9), it seemed possible that these fluctuations might represent reactions to minor traumata not perceptible to the examiner-possibly even of an unconscious nature. To test this hypothesis, three subjects, two of whom had received no sedation, were subjected to a sudden, very loud noise, 120 seconds after the sampling had started. The results of this experiment are shown in the lower part of Table V. In one subject (R. W.) a welldefined "spike" occurred 120 seconds after the noise; in the other two subjects no such response 


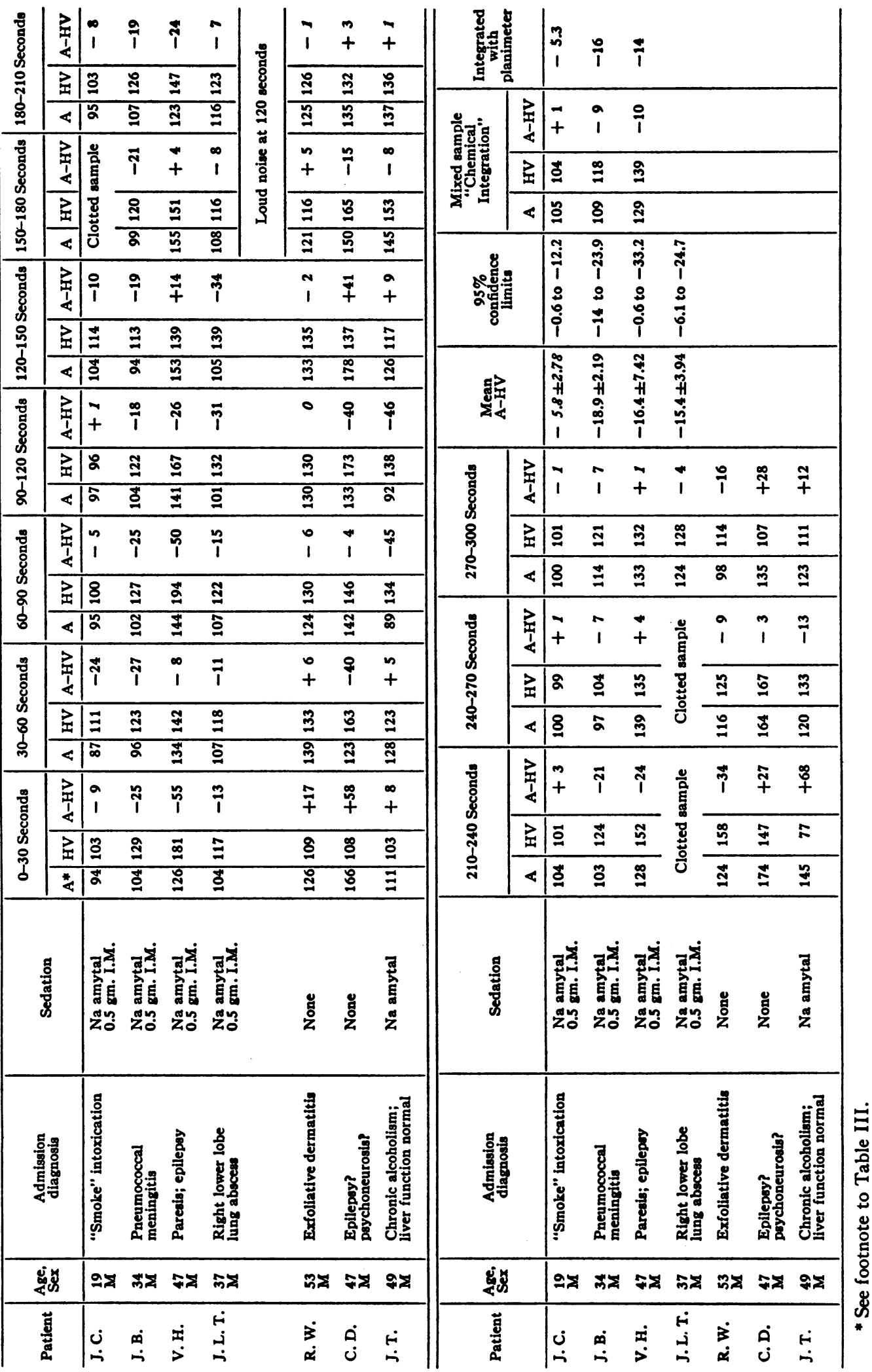


occurred. It is noteworthy that C. D. had widely fluctuating $\mathrm{A}-\mathrm{HV}$ values during the entire procedure, in spite of the fact that he did not appear anxious at any time.

\section{DISCUSSION}

This study has evaluated the variations in glucose production by the splanchnic system of human beings under experimental circumstances which protect them, as far as possible, from disturbing influences. The subjects were fasting, resting in a quiet environment and (except in two special cases) sedated to the point of moderate drowsiness. All manipulations were performed under local anesthesia, and pain stimuli were practically absent. Psychic stimulation arising from the strangeness of the environment, resentment at missing breakfast, etc., were undoubtedly present. It is impossible to evaluate the role which these factors played in disturbing the experimental subjects, but there can be no doubt that emotional influences may have been very important. It seems probable, however, that their effect was the least which could be achieved in this type of study.

In spite of the favorable environment and relative lack of overt stimulation, the observations reported above show clearly that rapid, unpredictable fluctuations occur in the splanchnic glucose balance, and that these variations are so large as to interfere seriously with attempts to estimate the mean balance over any protracted period of time.

The changes in splanchnic glucose balance are probably the results of at least three factors. The first and probably least important is experimental error. The data pertaining to the accuracy of the glucose determination indicate that only a very small portion of the variation could be a result of errors in this estimation.

A second cause of variation is a result of changes in the splanchnic blood flow. The fluctuations in the hepatic blood flow as estimated by the methods available are considerable (coefficient of variation $12.4 \%$ ) but since one cannot obtain true duplicate determinations, it is impossible to estimate how much of the variation is due to errors of analysis and how much to actual physiological alterations in the blood flow. In any case, variations of splanchnic balance of the order of 15 or $20 \%$ may be accounted for by fluctuations of splanchnic blood flow.

The studies of the A-HV difference, however, show that even this source of variation is relatively minor in comparison with the rapid changes in splanchnic A-HV glucose differences. It seems probable that most of the change observed in the value of $\mathrm{A}-\mathrm{HV}$ is due to actual variations in the rate of production of glucose by the liver and in the rate of utilization of glucose by the gut.

It has been shown that variations in the arterial glucose concentration can cause variations in the splanchnic glucose balance (3). In the present study, the arterial glucose concentration was not manipulated experimentally and remained fairly constant. In no case did it reach levels of definite hyper- or hypoglycemia. It seems unlikely therefore that the rapid variations of $\mathrm{A}-\mathrm{HV}$ glucose difference were a response to alterations of the arterial glucose concentration.

Hinkle (9) has shown that rapid alterations in the venous glucose concentration can be produced by psychic stimulation. The importance of this factor was not investigated directly in the present study, except in three subjects who were startled suddenly by a loud noise. This type of stimulus is admittedly a naive one, since its psychological significance will vary from one subject to another. Moreover, the effect of such an extraneous stimulus might be simply to distract the subject from his more significant reveries. The advantage of the single stimulus, however, in making possible accurate timing of the response, more than outweighed the psychiatric disadvantages in the present study. In one of these subjects, a "spike" of glucose output occurred after about the time interval which might have been expected if it resulted from the release of epinephrine. The other two subjects showed no such response. These observations indicate, at best, that "startle" reactions might be able to produce fluctuations comparable to those seen spontaneously. There is no direct evidence, however, that this type of reaction is actually involved in the production of the fluctuating A-HV difference.

The variability of the splanchnic glucose balance under rigidly controlled "basal" conditions raises grave doubts whether these observations have any relationship to the normal splanchnic glu- 
cose balance under the vicissitudes of daily life. The momentary fluctuations observed under these specially protected conditions are probably negligible in comparison with the variations induced by ordinary living. It is therefore questionable whether the estimations obtained by liver-catheterization techniques have any validity as representations of the daily 24-hour balances. Although these estimations have, in the past, been used as the basis for calculating the metabolic mixture and glucose utilization $(3,4)$, no confidence can be given to such calculations because (1) the estimates of A-HV have been based upon too few observations, and (2) even with many more observations, the value for $\mathrm{A}-\mathrm{HV}$ (and therefore for splanchnic balance) would have meaning only with regard to the moments during which the estimation was being made.

\section{SUMMARY AND CONCLUSIONS}

The arterio-hepatic venous glucose difference and hepatic blood flow have been estimated at intervals varying from 30 seconds to 10 minutes in 11 normal male human subjects under basal conditions. There is a very great spontaneous (and probably physiological) variation in the splanchnic glucose balance. In view of this unpredictable fluctuation, it is doubtful that estimates of splanchnic glucose balance are valid for any periods other than the actual time of sampling. Moreover, unless constant sampling techniques are used, the estimate of the splanchnic balance is valid only for the instant during which blood was actually being withdrawn.

In view of these findings, it seems unwise to use data obtained by the liver-catheter technique to estimate overall metabolic balances or rates of glucose utilization.

\section{REFERENCES}

1. Mann, F. C., and Magath, T. B., Studies on the physiology of the liver. II. The effect of the removal of the liver on the blood sugar level. Arch. Int. Med., 1922, 30, 73.

2a. Soskin, S., Essex, H. E., Herrick, J. F., and Mann, F. C., The mechanism of regulation of the blood sugar by the liver. Am. J. Physiol., 1938, 124, 558.

b. Lipscomb, A., and Crandall, L. A., Jr., Hepatic blood flow and glucose output in normal unanesthetized dogs. Am. J. Physiol., 1947, 148, 302.

3. Bondy, P. K., James, D. F., and Farrar, B. W., Studies of the role of the liver in human carbohydrate metabolism by the venous catheter technic. I. Normal subjects under fasting conditions and following the injection of glucose. J. Clin. Invest., 1949, 28, 238.

4a. Bondy, P. K., Bloom, W. L., Whitner, V. S., and Farrar, B. W., Studies of the role of the liver in human carbohydrate metabolism by the venous catheter technic. II. Patients with diabetic ketosis, before and after the administration of insulin. J. Clin. Invest., 1949, 28, 1126.

b. Myers, J. D., Net splanchnic glucose production in normal man and in various disease states. J. Clin. Invest., 1950, 29, 1421.

5. Somogyi, M., The use of copper and iron salts for the deproteinization of blood. J. Biol. Chem., 1931, 90, 725.

6. Bradley, S. E., Ingelfinger, F. J., Bradley, G. P., and Curry, J. J., The estimation of hepatic blood flow in man. J. Clin. Invest., 1945, 24, 890.

7. Nelson, N., A photometric adaptation of the Somogyi method for the determination of glucose. J. Biol. Chem., 1944, 153, 375.

8. Snedecor, G. W., Statistical Methods Applied to Experiments in Agriculture and Biology. Iowa State College Press, Ames, Iowa, 1948, 4th Ed.

9a. Hinkle, L. E., Jr., and Wolf, S., Variations in blood glucose in diabetes mellitus. J. Clin. Invest., 1951, 30, 649.

b. Hinkle, L. E., Jr., Conger, G. B., and Wolf, S., Studies on diabetes mellitus: the relation of stressful life situations to the concentration of ketone bodies in the blood of diabetic and non-diabetic humans. J. Clin. Invest., 1950, 29, 754. 